\title{
FATIGUE CRACKS DETECTION USING PZT TRANSDUCERS UNDER THE INFLUENCE OF UNCERTAIN ENVIRONMENTAL FACTORS
}

\author{
Michal Dziendzikowski \\ Slawomir Klimaszewski \\ Krzysztof Dragan \\ Air Force Institute of Technology, Ks. Boleslawa 6 street, 01-494 Warsaw, Poland \\ michal.dziendzikowski@itwl.pl, slawomir.klimaszewski@itwl.pl, krzysztof.dragan@itwl.pl
}

\begin{abstract}
This paper presents technique for qualitative assessment of fatigue crack growth monitoring, utilizing guided elastic waves generated by the sparse PZT piezoelectric transducers network in the pitch - catch configuration. Two Damage Indices (DIs) correlated with the total energy received by a given sensor are used to detect fatigue cracks and monitor their growth. The indices proposed carry marginal signal information content in order to decrease their sensitivity with respect to other undesired non-controllable factors which may distort the received signal. The reason for that is to limit the false calls ratio which besides the damage detection capability of a system, plays a crucial role in applications. However, even such simplified damage indices can alter over a long term, leading to the misclassification problem. Considering a single sensing path, it is very difficult to distinguish whether the resultant change of DIs is caused by a damage or due to decoherence of these DIs. Therefore, assessment approaches based on threshold levels fixed separately for DIs obtained on each of the sensing paths, would eventually lead to a false call. An alternative approach is to compare changes of DIs for all sensing paths. Developing damage distorts the signal only for the sensing paths in its proximity. In order to decrease the misclassification risk, a method of compensating such DIs drift is proposed. The main features and damage detection capabilities of this method will be demonstrated by conducting a laboratory fatigue test of an aircraft panel. The proposed approach has been verified on a real structure during fatigue test of a helicopter tail boom.
\end{abstract}

Keywords: Structural Health Monitoring, PZT transducers, fatigue cracks detection, environmental factors compensation.

\section{INTRODUCTION}

Over 40 years have passed since introducing the damage tolerance philosophy into the design process of aircraft [1]. One of the pillars of an aircraft's structural integrity is controlling the assumed loads spectrum and its state. The loads that act on a structure determine intervals between subsequent non-destructive inspections (NDI), which provide information about the condition of the aircraft structure. The way that particular aircraft is operated after its introduction into service does not necessarily suit to its pre-assumed profile, thus the scheduled timeline of NDI may be no longer appropriate. In the past, this contributed to flight accidents, e.g. the accident of Boeing 737 of Aloha Airlines in 1988, which was subjected to an excessive number of cabin pressurizing cycles, accelerating the development of WFD (Widespread Fatigue Damage) [2]. In order to overcome the abovementioned drawbacks of classical procedures, for over 25 years an intensive 
research activity, focused on developing Structural Health Monitoring (SHM) systems, has been conducted. The SHM systems are intended to be used for the purpose of continuous, on-line monitoring of the aircraft structure. The application of such systems would definitely enhance safety, especially when considering hardly accessible 'hot-spots', but it could also save up to 50\% of necessary inspections depending on the aircraft type [2]. One of the approaches to SHM is to use a PZT transducers network deployed on a monitored structure. In this approach, the idea of damage detection relies on comparising the acquired signals for the initial and the damaged state of the structure [2-5]. Elastic waves, actuated by PZT transducers, can propagate significant distances and are sensitive to local structure discontinuities and deformations, providing a tool for detecting local damage of aerospace structures, irrespectively of the materials being used for their manufacturing.

\section{THE METHODOLOGY OF THE APPROACH}

Structural damage can result in observable changes of the signal generated by the network sensors. The state of a monitored structure is assessed based on the chosen signal characteristics called the Damage Indices (DIs). The acquired signals can be also affected by factors other than damages thus posing the risk of false indications. Therefore, DIs used for the structure assessment needs to be balanced between the sensitivity to damages and the stability under varying working conditions of transducers. In the adopted approach, the DIs carry marginal signal information content. Denoted as $f_{g s}^{e n v}$ the envelope of a signal generated by the transducer $g$ and received by the sensor $s$ and as $f_{g s, b}^{e n v}$ the envelope of the corresponding baseline, i.e. the reference signal obtained for the initial state of the structure, the Damage Index used in this paper is given as follows [6]:

$$
D I(g, s)=1-r_{f_{g s}^{e n v} f_{s s, b}^{e n v}}
$$

where $r_{x y}$ stands for the sample correlation of series $x, y$. The DI is correlated with the total energy received by a given sensor and also with its distribution in time during the measurement. Thus it is sensitive to the two main modes of the guided wave's interaction with a fatigue crack, i.e. its transmission and reflection from a damage.

However, damage indices can alter over a long term, due to the influence of factors other than damage on PZT transducers, e.g. temperature changes or their aging, which leads to the misclassification problem. Considering a single sensing path, it is very difficult to distinguish whether the resultant change of DI is caused by a damage or due to such a decoherence. A developing damage of finite extent should distort the signal only for the sensing paths running in its proximity. In order to decrease the misclassification risk, a method of compensating such DIs' drift can be used, for which the compensated DI is given by the formula [6]:

$$
D I_{\text {comp }}(g, s)=D I(g, s)-m-(m(g)-m)-(m(s)-m),
$$

where

$$
m=\min _{g, s} D I(g, s)
$$

is the minimum of DIs considering all of the network's sensing paths and 


$$
m(p)=\min _{t} D I(p, t), \quad p=g, s
$$

is the minimum of DIs for sensing paths originating from a given transducer $p$. The first subtracted term $-m$ in the formula (2) corresponds to homogeneous DIs flow cancelation, whereas the last two terms suppress the signal decoherence inhomogeneities of the generator $g$ and the sensor $s$.

\section{EXPERIMENTAL VERIFICATION OF THE APPROACH}

The described approach was used for the purpose of fatigue testing of a helicopter's tail boom, which was part of the ASTYANAX (Aircraft fuselage crack monitoring sYstem And prognosis through on-boArd eXpert sensor network) project governed by the European Defence Agency. The platform used for the project was Mi-8/17 helicopter. The first part of the project was simulation of hard landing which in particular allowed tests of new auxetic foams for pilot seats [7].

The tail boom of the helicopter was equipped with FBG, PZT sensors as well as crack gauges. In Figure 1, a PZT network and crack gauges installed near an introduced crack are presented.

Two types of PZT transducers were used: single- and multi-layered. The following parameters of PZT excitation were used:

- excitation frequency [kHz]: $100,150,200,250,300$;

- duration: 3 or 8 periods;

- window type: Hanning.

The signal from the PZT network is gathered under varying loads which can contribute to the DIs drift effect.

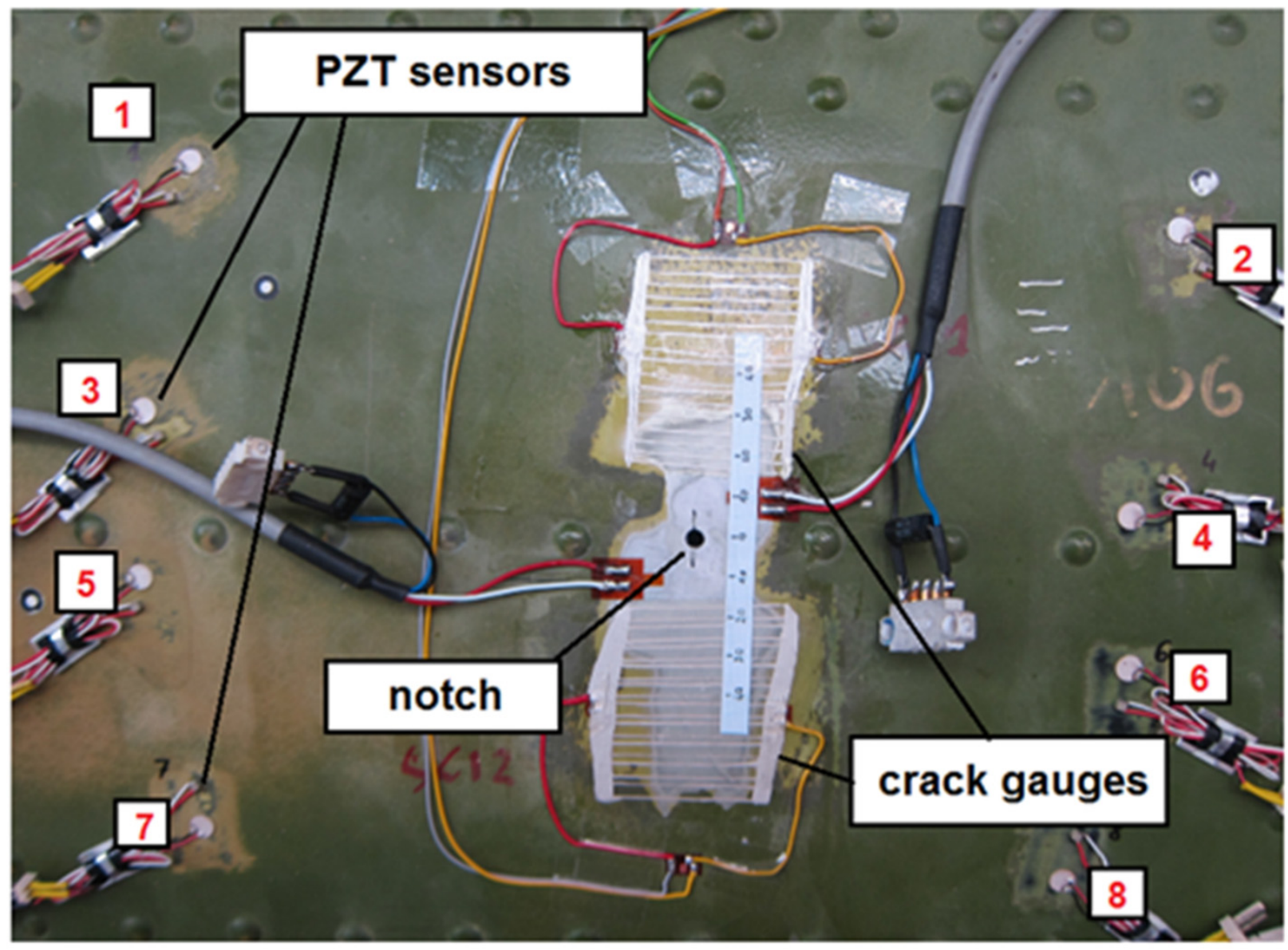

Fig. 1. PZT sensors and crack gauges deployed on the helicopter structure with numeration of PZT network 
In order to illustrate DI change within a network, a map:

$$
I^{\max }(x, y)=\max _{g, s} I(g, s)
$$

is used, where

$$
I_{g s}(x, y)=D I(g, s) R_{g s}(x, y), \quad R_{g s}(x, y)=\left(\frac{l_{g s}}{l_{g p}+l_{g s}}\right)^{40}
$$

for $l_{g p}, l_{g s}$ denoting the distance of the point $p=(x, y)$ from the generator $g$ or the sensor $s$ respectively, and $l_{g s}$ is the distance between generator $g$ and sensor $s$. The DI value on a given sensing path is weighted with a map $R_{g s}$ representing the geometry of a given sensing path. Then for a point $p$, the maximum value of $I_{g s}$ at that point is adopted for the value of $I^{\max }$.

Figure 2 shows the map (9) before (Fig. 2(a)) and after (Fig. 2(b)) the compensation of the DI for single layered transducers at the frequency $150 \mathrm{kHz}$. The change in the compensated DIs is the highest for the sensing paths running in close proximity of the crack (Fig. 2(b)), whereas uncompensated DIs can change also for paths for which the signal should not be influenced by a damage (Fig. 2(a)), which can lead to the false indication of the system.
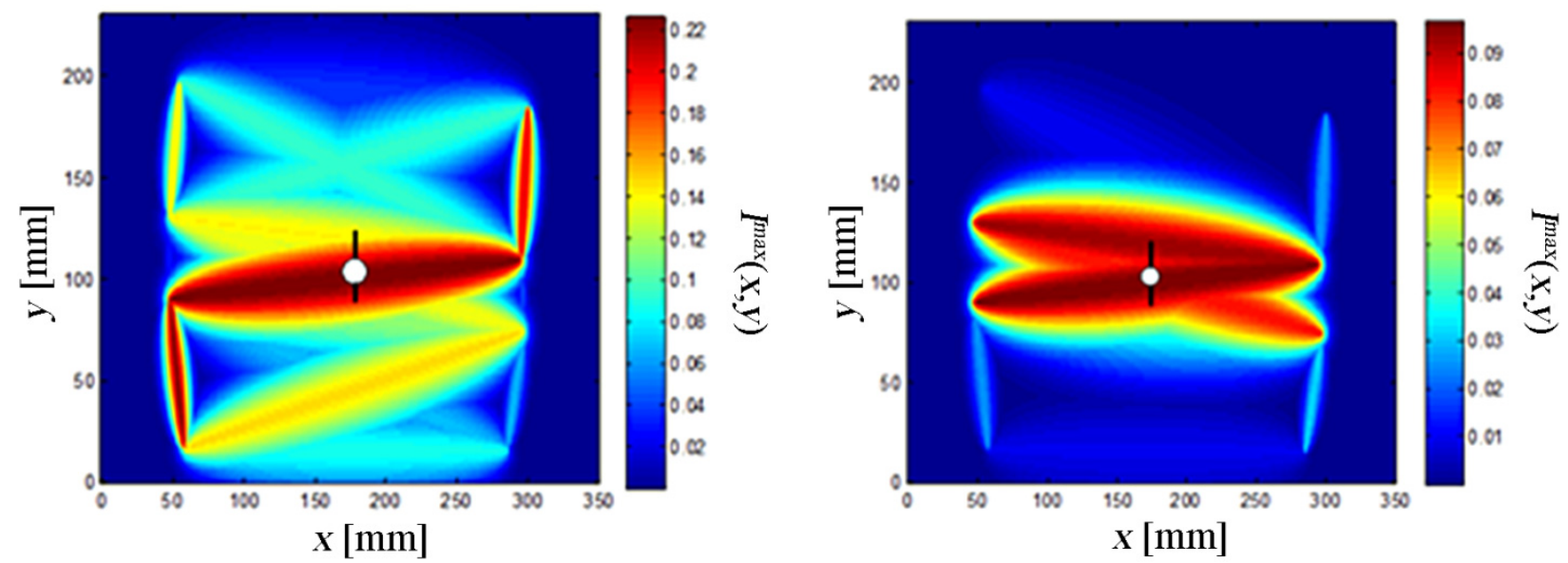

Fig. 2. Visualisation of Damage Index change within the network using: (a) not compensated and (b) compensated DI

\section{SUMMARY}

In the paper, a method of monitoring fatigue crack growth by means of elastic guided waves was presented. The crack growth resolution of the approach is restricted to indicate if sensing paths of the PZT sensor network were intersected by a developing crack. This is due to limited information content carried by the Damage Indices used. In return, the DIs exhibit small sensitivity to signal decoherence in time. This makes it possible to apply the proposed DIs drift compensation formula, and, consequently, decrease significantly false positive indications, which, besides the damage detection capability, is one of the crucial factors in SHM applications. The approach was validated using data collected during fatigue tests of a helicopter's tail boom.

\section{Acknowledgments}

The work presented in this paper has been supported by the European Defence Agency (EDA) through Contract No B 1288 ESM2 GP entitled "Aircraft Fuselage Crack Monitoring System and Prognosis through on-board Expert Sensor Network (ASTYANAX)". The tri-national 
ASTYANAX project involves the following partners: Politecnico di Milano (consortium leader), Alenia Aermacchi and Agusta Westland (Italy), Air Force Institute of Technology, Military Aviation Works No. 1 and AGH University of Science and Technology (Poland), and Instituto Nacional de Técnica Aeroespacial (Spain).

\section{REFERENCES}

[1] Osgood, C. (1982). Fatigue Design - 2nd edition. Pergamon Press.

[2] Staszewski, W., Boller, C., Tomlinson, G. R. (2004). Health monitoring of aerospace structures: smart sensor technologies and signal processing, John Wiley \& Sons.

[3] Giurgiutiu, V. (2014). Structural health monitoring: with piezoelectric wafer active sensors 2nd ed., Academic Press.

[4] Stepinski, T., Uhl, T., Staszewski, W. (2013). Advanced Structural Damage Detection: From Theory to Engineering Applications, John Wiley \& Sons.

[5] Su, Z., \& Ye, L. (2009). Identification of damage using Lamb waves: from fundamentals to applications, Springer.

[6] Dragan, K. \& Dziendzikowski, M. (2016). Structural Health Monitoring 15, pp. 423-437.

[7] Lisiecki, J., Blazejewicz, T., Klysz, S., Gmurczyk, G., Reymer, P., Mikulowski, G. (2013). Tests of polyurethane foams with negative Poisson's ratio, Physica Status Solidi (B) 250, pp. 1988-1995. 